\title{
Exigências Térmicas e Potencial de Desenvolvimento dos Parasitóides Telenomus podisi Ashmead e Trissolcus brochymenae (Ashmead) em ovos do Percevejo Predador Podisus nigrispinus (Dallas)
}

\author{
Jorge B. Torres ${ }^{1}$, Dirceu Pratissoli e José C. Zanuncio ${ }^{3}$ \\ ${ }^{1}$ Departamento de Agronomia e Fitossanidade, Universidade Federal Rural de Pernambuco, \\ Av. Dom Manoel de Medeiros, s/n, Dois Irmãos, 52171-900, Recife, PE. \\ ${ }^{2}$ Clínica Fitossanitária, Centro Agropecuário da Universidade Federal do \\ Espírito Santo, 29500-000, Alegre, ES. \\ ${ }^{3}$ Departamento de Biologia Animal, Universidade Federal de Viçosa, \\ 36571-000, Viçosa, MG.
}

An. Soc. Entomol. Brasil 26(3): 445-453 (1997)

Thermal Requirements and Development of the Egg Parasitoids Telenomus podisi Ashmead and Trissolcus brochymenae (Ashmead) on the Predator

Stinkbug Podisus nigrispinus (Dallas)

\begin{abstract}
Development and viability of the egg parasitoids Telenomus podisi Ashmead and Trissolcus brochymenae (Ashmead) (Hymenoptera: Scelionidae) were studied at five constant temperatures ranging from $17^{\circ} \mathrm{C}$ to $32^{\circ} \mathrm{C}$ on its host, the predatory bug Podisus nigrispinus (Dallas) (Heteroptera: Pentatomidae). Female development from egg to adult ranged from 10.8 days $\left(32^{\circ} \mathrm{C}\right)$ to 48.6 days $\left(17^{\circ} \mathrm{C}\right)$ for $T$. podisi, and from 10.2 days $\left(32^{\circ} \mathrm{C}\right)$ to 35.4 days $\left(17^{\circ} \mathrm{C}\right)$ for $T$. brochymenae. Parasitism viability ranged from 89.0 to $92.7 \%$ for $T$. brochymenae. However, T. podisi viability decreased in lower and higher temperatures. Lower threshold temperatures for egg to adult female development was estimated at $11.1^{\circ} \mathrm{C}$ for T. podisi and $9.5^{\circ} \mathrm{C}$ for $T$. brochymenae. Thermal requirements for this period was 205.3 and 214.7 degree-days for $T$. podisi and $T$. brochymenae, respectively. Number of generations per year of these parasitoids and its host, considering the temperature conditions of eucalyptus areas, were estimated to be 22.6, 23.9 and 10.2 for $T$. podisi, $T$. brochymenae and $P$. nigrispinus, respectively. These results indicate that $T$. brochymenae is better adapted to temperature variations than $T$. podisi, however, both species can successfully parasitize $P$. nigrispinus.
\end{abstract}

KEY WORDS: Insecta, Scelionidae, predatory bug, biological control, temperature.

RESUMO - O desenvolvimento e a viabilidade dos parasitóides de ovos Telenomus podisi Ashmead e Trissolcus brochymenae (Ashmead) (Hymenoptera: Scelionidae) foram estudados em cinco temperaturas constantes de 17 a $32^{\circ} \mathrm{C}$, em seu hospedeiro, o percevejo predador Podisus nigrispinus (Dallas) (Heteroptera: Pentatomidae). A duração do período ovo-adulto das fêmeas foi de 10,8 dias $\left(32^{\circ} \mathrm{C}\right)$ a 48,6 dias $\left(17^{\circ} \mathrm{C}\right)$ para $T$. podisi e de 10,2 dias $\left(32^{\circ} \mathrm{C}\right)$ a 35,4 dias $\left(17^{\circ} \mathrm{C}\right)$ para $T$. brochymenae. A viabilidade, em todas as temperaturas, manteve-se entre 89,0 e $92,7 \%$ para $T$. brochymenae, com redução significativa 
da viabilidade de T. podisi nas temperaturas extremas de 17 e $32^{\circ} \mathrm{C}$. A temperatura base e a constante térmica, para o desenvolvimento das fêmeas foram de $11,1^{\circ} \mathrm{C}$ e $9,5^{\circ} \mathrm{C}$ e de 205,3 e 214,7 graus dias, para $T$. podisi e $T$. brochymenae, respectivamente. $\mathrm{O}$ número de gerações por ano, para os parasitóides e o hospedeiro, estimado com base nas temperaturas de áreas de cultivo de eucalipto, foram de 22,6; 23,9 e 10,2, para $T$. podisi, $T$. brochymenae e $P$. nigrispinus, respectivamente. Essas observações indicam que T. brochymenae é melhor adaptado a variações de temperaturas que $T$. podisi, no entanto, as duas espécies parasitam com sucesso $P$. nigrispinus.

PALAVRAS-CHAVE: Insecta, Scelionidae, percevejo predador, controle biológico, temperatura.

O emprego de modelos matemáticos, para previsão de ocorrência de pragas e de seus inimigos naturais, é composto por vários elementos, incluindo os climáticos e, em especial, a temperatura (Higley et al. 1986). Esta afeta, diretamente, o desenvolvimento dos insetos, sendo empregada para o cálculo de graus-dia, na previsão de eventos biológicos, controlados pelo acúmulo de calor (Miller 1992). Assim, entre muitos fatores afetando os inimigos naturais, a adaptação térmica é uma das mais fáceis de ser medida e é essencial para o sucesso e estabelecimento desses agentes (Frazer \& McGregor 1992), sendo que, através da constante térmica $(K)$ e da temperatura base $(\mathrm{tb})$, pode-se manipular a criação massal de inimigos naturais, com previsão para suas liberações.

O percevejo predador Podisus nigrispinus (Dallas) (Heteroptera: Pentatomidae), em diversos agroecossistemas, é considerado como importante no controle de lagartas desfolhadoras e outros insetos, tanto quanto sua ocorrência natural ou seu emprego em liberações programadas (Zanuncio et al. 1994, Torres et al. 1996a). Suas populações nos agroecossistemas podem ser afetadas por muitos fatores, como a disponibilidade de presas (O’Neil \& Stimac 1988) e a capacidade de defesa (Tostowark 1972), por parasitóides de ovos, que podem atingir níveis significativos em relação aos demais fatores de mortalidade de ovos (J.B. Torres, dados não publicados) e, certamente, pela variação da temperatura.

Os parasitóides de ovos Telenomus podisi Ashmead e Trissolcus brochymenae (Ashmead) (Hymenoptera: Scelionidae) têm sido encontrados, em diversos agroecossistemas, parasitando inúmeros Pentatomidae (Barcelos et al. 1994, Corrêa-Ferreira \& Moscardi 1995, Torres et al. 1996b), mostrando, de certo modo, sua adaptação a diferentes hospedeiros e condições de clima. Entre as espécies de ocorrência natural sobre P. nigrispinus, T. podisi e T. brochymenae tem se mostrado melhor adaptadas a este hospedeiro, apresentando maiores taxas líquida de reprodução e intrínsica de crescimento do que outras espécies, como Ooencyrtus sp. (Hymenoptera: Encyrtidae) (Torres et al. 1996c).

Este trabalho objetivou estudar o desenvolvimento e determinar as exigências térmicas dos parasitóides de ovos T. podisi e T. brochymenae. Também, estimar o número provável de gerações desses parasitóides e do percevejo predador e hospedeiro, baseado nas condições de campo, onde têm sido realizadas liberações do predador e constatadas a ocorrência de parasitismo de ovos.

\section{Material e Métodos}

Obtenção dos Parasitóides. T. podisi e $T$. brochymenae foram coletados em áreas de 
surto de lagartas desfolhadoras de eucalipto, onde estavam sendo realizadas liberações do predador P. nigrispinus (Torres et al. 1996b). As colônias dos parasitóides foram mantidas em laboratório, parasitando ovos de $P$. nigrispinus, em tubos de ensaio de 2,5 x 10 $\mathrm{cm}$, tampados com algodão e tendo mel de abelha como alimento. Esses tubos foram mantidos em suporte, na horizontal, em câmaras a $25 \pm 1^{\circ} \mathrm{C}, 65 \pm 5 \%$ de UR e $14 \mathrm{~h}$ de fotofase.

\section{Determinação da Temperatura Base (tb) e} Constante Térmica (K). Foram utilizadas duas cubas de vidro de $0,20 \mathrm{~cm}^{3}$ (uma para cada espécie de parasitóide), tendo em cada uma delas 25 posturas de $P$. nigrispinus, com 15 a 30 ovos de idade inferior a $24 \mathrm{~h}$. As posturas foram submetidas, durante seis horas, ao parasitismo por fêmeas de T. podisi ou $T$. brochymenae acasaladas e com um dia de idade. Em seguida, as posturas parasitadas, foram transferidas para tubos de ensaio de 2,5 x $10 \mathrm{~cm}$ e tranferidas para câmaras com temperaturas de $17,20,25,28$ e $32^{\circ} \mathrm{C}$, mantendo-se cinco posturas por espécie de parasitóide, para cada temperatura (repetições). Diariamente, as posturas foram vistoriadas, para a remoção de ninfas do predador, pois estas, no segundo estádio, predam os demais ovos. Os ovos que não deram origem a ninfas e tornaram-se escuros foram computados, para o cálculo da viabilidade dos ovos parasitados.

O período ovo-adulto para machos e fêmeas, razão sexual (número de fêmeas/ número de fêmeas + número de machos) e viabilidade dos ovos parasitados (percentagem de emergência) foram determinadas para T. podisi e T. brochymenae, nas diferentes temperaturas. A viabilidade dos ovos foi calculado baseado no número de ovos considerados parasitados, devido ao escurecimento dos mesmos com o desenvolvimento dos parasitóides e a emergência de adultos. Estes foram sexados, com auxílio de microscópio estereoscópico, pelo dimorfismo das antenas (Morril 1907).

A temperatura base (tb) e a constante témica (K) dos parasitóides T. podisi e $T$. brochymenae e $P$. nigrispinus foram estimadas pela regressão linear simples $\mathrm{y}=\mathrm{a}$ $+b_{t}$, sendo $y$ a duração, em dias, da oviposição à emergência dos adultos nas respectivas temperaturas $t\left({ }^{\circ} \mathrm{C}\right)$. A tb foi obtida extrapolando os valores da equação para o ponto que corta o eixo $x\left(\mathrm{tb}=-\mathrm{a}_{1} / \mathrm{b}_{1}\right)$. O valor de $\mathrm{K}$ foi estimado em relação à tb requerida para completar o desenvolvimento, pelo valor recíproco do coeficiente linear da equação (K $=1 / \mathrm{b}_{\mathrm{t}}$ ), de acordo com Campell et al. (1974). Para o percevejo predador, foi considerado o período de incubação dos ovos, da fase de ninfa e de pré-oviposição, nas temperaturas de $18,20,25,30,32$ e $35^{\circ} \mathrm{C}$ (Didonet et al. 1996).

Estimativa do Número de Gerações. O número provável de gerações, por mês e por ano, para fêmeas de $T$. podisi e $T$. brochymenae e de $P$. nigrispinus, foi calculado baseando-se nas exigências térmicas (tb e K) e das temperaturas médias mensais e anuais de 1988 a $1993\left(\mathrm{t}_{\mathrm{c}}\right)$, em áreas de reflorestamento com eucalipto no cerrado de Minas Gerais, através da equação: $\mathrm{NG}=$ $\left\{\mathrm{T} /\left[\mathrm{k}\left(\mathrm{t}_{\mathrm{c}}-\mathrm{tb}\right)\right]\right\}$, onde T é o tempo considerado em mês (30 dias) ou ano (365 dias) e os demais parâmetros definidos anteriormente. As temperaturas médias $\left(\mathrm{t}_{\mathrm{c}}\right)$, usadas para as estimativas, foram obtidas considerando-se a média ponderada entre as temperaturas médias do dia e da noite, empregando-se para esse cálculo a fotofase de $14 \mathrm{~h}$, utilizada no estudo de determinação da temperatura base (tb) e constante térmica (K), em laboratório.

Os resultados de duração e viabilidade do ciclo ovo-adulto, para machos e fêmeas, além da razão sexual dos parasitóides, foram submetidos à análise de variância, e as médias comparadas pelo teste de Tukey, a 5\% de probabilidade.

\section{Resultados e Discussão}

A duração do período ovo-adulto (Tabela 1) decresceu com o aumento da temperatura até $32^{\circ} \mathrm{C}(P<0,05)$. Entretanto, observa-se 
Tabela 1. Duração média do ciclo (ovo-adulto), viabilidade e razão sexual de Telenomus podisi e Trissolcus brochymenae, criados em ovos de Podisus nigrispinus, em diferentes temperaturas.

\begin{tabular}{|c|c|c|c|c|c|}
\hline \multirow{2}{*}{$\begin{array}{l}\text { Temperatura } \\
\left({ }^{\circ} \mathrm{C}\right) \\
\end{array}$} & \multicolumn{3}{|c|}{ Duração Média $( \pm \mathrm{DP})^{1}$ e Int. de Variação (dias) } & \multirow{2}{*}{$\frac{\text { Viabilidade }^{1}}{(\%)}$} & \multirow{2}{*}{$\begin{array}{l}\text { Razão } \\
\text { Sexual }^{1,2} \\
\end{array}$} \\
\hline & \multicolumn{2}{|c|}{ Fêmea } & Macho & & \\
\hline \multicolumn{6}{|c|}{ Telenomus podisi } \\
\hline 17 & $48,6 \pm 6,2 \mathrm{a}$ & $(45,0$ & $43,7 \pm 1,5$ a $(43,0-45,0)$ & $44,3 \pm 26,9 b$ & $0,85 \mathrm{a}$ \\
\hline 20 & $21,8 \pm 0,1 \mathrm{~b}$ & $(21$, & $20,0 \pm 0,6 \mathrm{~b} \quad(19,4-$ & $95,2 \pm$ & $0,88 \mathrm{a}$ \\
\hline 25 & $12,7 \pm 0,6 \mathrm{c}$ & $3,5)$ & $0,4 \mathrm{c} \quad(11,0-12,0)$ & $90,1 \pm 10,5 \mathrm{a}$ & $0,86 \mathrm{a}$ \\
\hline 28 & $11,6 \pm 0,4 \mathrm{c}$ & $(11,0$ & $9,9 \pm 0,6 \mathrm{~d} \quad(9,0-10,8)$ & $91,1 \pm 6,4$ a & $0,89 \mathrm{a}$ \\
\hline 32 & $10,8 \pm 0,6 \mathrm{c}$ & $(10,0-11,7)$ & $9,6 \pm 0,6 \mathrm{~d}$ & $59,8 \pm 18,7 \mathrm{~b}$ & $0,87 \mathrm{a}$ \\
\hline \multicolumn{6}{|c|}{ Trissolcus brochymenae } \\
\hline 17 & $35,4 \pm 0,7 \mathrm{a}$ & $(34,3-36,1)$ & $31,8 \pm 1,8$ a $\quad(30,5$ & $92,7 \pm 4,9$ & $0,90 \mathrm{a}$ \\
\hline 20 & $19,1 \pm 0,1 b$ & $(19,0-19,7)$ & $17,3 \pm 0,3 b \quad(17,0-17,8)$ & $90,0 \pm 9,4 \mathrm{a}$ & $0,88 \mathrm{a}$ \\
\hline 25 & $13,0 \pm 0,0 \mathrm{c}$ & $(13,0-13,0)$ & $12,0 \pm 0,0 \mathrm{c} \quad(12,0-12,0)$ & $89,0 \pm 10,4 \mathrm{a}$ & a 0,89 a \\
\hline 28 & $11,1 \pm 0,2 \mathrm{~d}$ & $(11,0-11,5)$ & $10,0 \pm 0,0 \mathrm{~d} \quad(10,0-10,0)$ & $92,3 \pm 7,2 \mathrm{a}$ & a 0,89 a \\
\hline 32 & $10,2 \pm 0,1 \mathrm{e}$ & $(10,0-10,4)$ & $(8,5-9,5)$ & $89,3 \pm 10,4 \mathrm{a}$ & $0,91 \mathrm{a}$ \\
\hline
\end{tabular}

${ }^{1}$ Médias \pm Desvio Padrão seguidas pela mesma letra, na coluna, não diferem estatisticamente pelo teste de Tukey a 5\% de probabilidade.

${ }^{2}$ Razão Sexual $=$ Fêmeas/Machos + Fêmeas.

que este decréscimo foi menos acentuado de 25 a $32^{\circ} \mathrm{C}$ do que de 17 a $25^{\circ} \mathrm{C}$ (Figs. 1, 2), para ambas as espécies de parasitóides. $\mathrm{O}$ escurecimento dos ovos, proveniente do desenvolvimento das larvas dos parasitóides e empregado para avaliação da viabilidade,

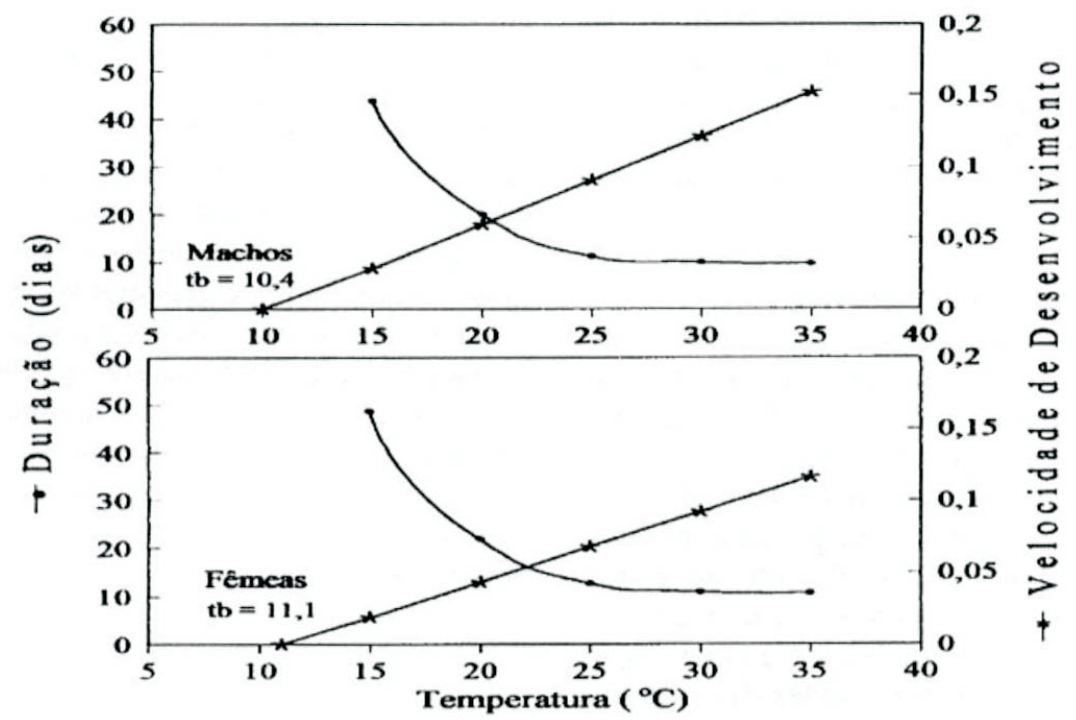

Figura 1. Desenvolvimento de machos e fêmeas de Telenomus podisi em ovos de Podisus nigrispinus, em diferentes temperaturas. 


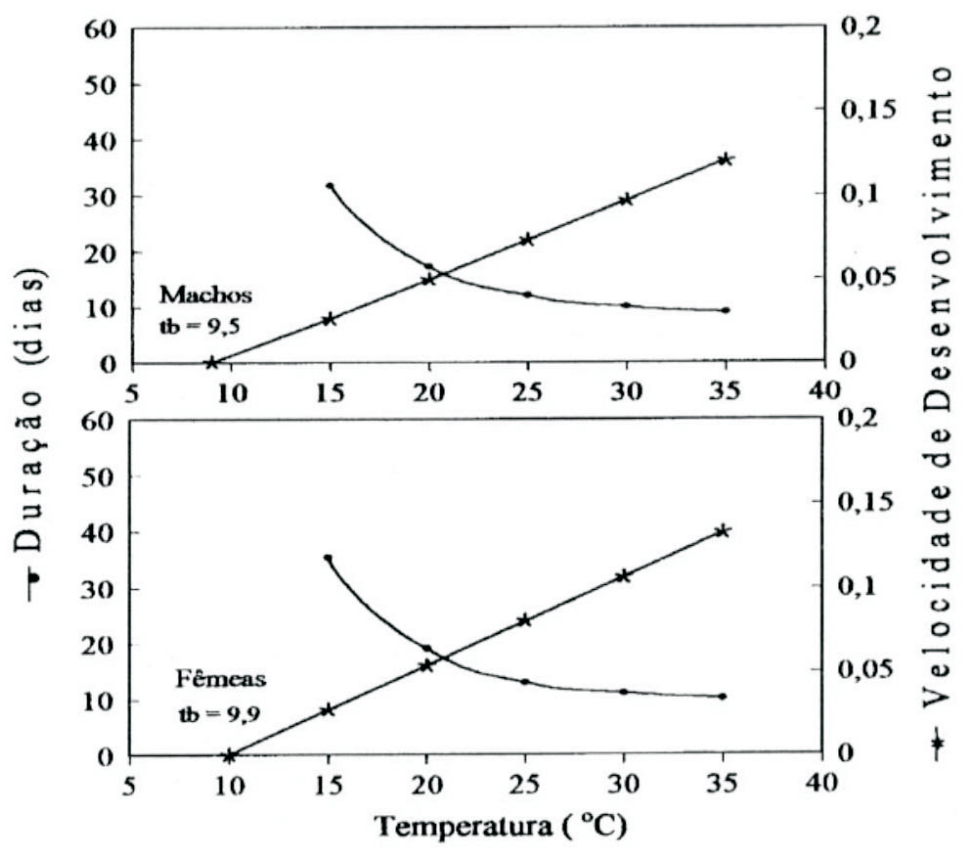

Figura 2. Denvolvimento de machos e fêmeas de Trissolcus brochymenae em ovos de Podisus nigrispinus, em diferentes temperaturas.

foi observado entre 3 a 4 dias para as temperaturas acima de $25^{\circ} \mathrm{C}$, chegando a 15 dias a $17^{\circ} \mathrm{C}$. Consequentemente, a $17^{\circ} \mathrm{C}$, o ciclo ovo-adulto apresentou maior variação para $T$. podisi, com amplitude do período de emergência de fêmeas e machos de 13 e 2 dias, respectivamente, e de 1,8 e 4,5 dias para $T$. brochymenae. No entanto, a $20,25,28$ e $32^{\circ} \mathrm{C}$, esta amplitude foi de 0,1 a 1,7 dias para $T$. podisi e de 0 a 0,7 dias para $T$. brochymenae.

O período ovo-adulto, para machos e fêmeas de $T$. podisi, foi de 9,6 e 10,8 dias a $32^{\circ} \mathrm{C}$ e, de 43,7 e 48,6 dias a $17^{\circ} \mathrm{C}$, enquanto para T. brochymenae foi de 9,0 e 10,2 e de 31,8 e 35,4 dias, respectivamente (Tabela 1), apresentando incremento de tempo de 0,8 ; 1,$9 ; 11,0$ e 37,8 dias para fêmeas de $T$. podisi e de 0,$9 ; 2,8 ; 8,9$ e 25,2 dias para as de $T$. brochymenae, caracterizando maior impacto da redução de temperatura sobre a primeira espécie. T. podisi exigiu 4,5 vezes mais tempo (ovo-adulto) a $17^{\circ} \mathrm{C} \mathrm{e}$, apenas, 2,0 vezes mais a $20^{\circ} \mathrm{C}$, com redução mais suave, do tempo requerido, a partir de $20^{\circ} \mathrm{C}$ (Fig. 1), enquanto $T$. brochymenae exigiu 3,5 e 1,9 vezes, nas mesmas temperaturas. T. brochymenae apresentou homogeneidade no decréscimo do tempo requerido, em todas as temperaturas, como é observado nas curvas de velocidade e duração de desenvolvimento dessa espécie (Fig. 2), comparado a T. podisi (Fig. 1).

A viabilidade dos ovos parasitados manteve-se entre 89,0 a 92,7\%, sem diferença significativa $(P>0,05)$ para $T$. brochymenae, mas $T$. podisi apresentou redução significativa $(P<0,05)$ nas temperaturas extremas de $17^{\circ} \mathrm{C}$ $(44,3 \%)$ e de $32^{\circ} \mathrm{C}(59,8 \%)$ (Tabela 1$)$. A temperatura de $17^{\circ} \mathrm{C}$ permitiu a obtenção de adultos de $T$. podisi, mas teve impacto significativo na sua emergência. Jubb \& Watson (1971) obtiveram resultados semelhantes para Trissolcus utahensis (Ashmead) a $15^{\circ} \mathrm{C}$ e Yeargan (1980) relatou que nenhum macho de $T$. podisi emergiu a 
Tabela 2. Temperatura base (tb), constante térmica $(\mathrm{K})$ e coeficiente de determinação $\left(\mathrm{R}^{2}\right)$ de machos e fêmeas de Telenomus podisi e Trissolcus brochymenae, criados em ovos de Podisus nigrispinus, em condições de laboratório.

\begin{tabular}{llcccc}
\hline Parasitóides/Predador & Sexo & $\begin{array}{c}\text { tb } \\
\left({ }^{\circ} \mathrm{C}\right)\end{array}$ & $\begin{array}{c}\mathrm{K} \\
(\mathrm{GD}) *\end{array}$ & $\begin{array}{c}\text { Equações de } \\
\text { Desenvolvimento }\end{array}$ & $\mathrm{R}^{2}$ \\
\hline Telenomus podisi & Fêmea & 11,1 & 205,3 & $1 / \mathrm{D}=-0,0540+0,00486 \mathrm{~T}$ & 92,12 \\
& Macho & 10,4 & 177,6 & $1 / \mathrm{D}=-0,0640+0,00617 \mathrm{~T}$ & 96,52 \\
& & & & & \\
Trissolcus brochymenae & Fêmea & 9,5 & 214,7 & $1 / \mathrm{D}=-0,0449+0,00473 \mathrm{~T}$ & 95,75 \\
& Macho & 9,9 & 189,2 & $1 / \mathrm{D}=-0,0522+0,00528 \mathrm{~T}$ & 97,12 \\
\hline
\end{tabular}

* Graus-dias

$15,5^{\circ} \mathrm{C}$, com somente $43,5 \%$ de emergência de fêmeas. A viabilidade de T. podisi a $17^{\circ} \mathrm{C}$ foi semelhante à obtida a $15^{\circ} \mathrm{C}$ por outros pesquisadores, trabalhando com insetos de clima temperado, indicando que esta espécie, proveniente de região de cerrado, onde ocorrem temperaturas mais elevadas, pode estar adaptada às condições tropicais.

A razão sexual dos parasitóides não foi afetada pela temperatura $(P>0,05)$, sendo de 0,88 a 0,91 para $T$. brochymenae, variando de um macho para 7,3 a 10,1 fêmeas e, entre 0,85 a 0,89 para $T$. podisi, com um macho para 5,7 a 8,1 fêmeas (Tabela 1). Esses resultados concordam com Waage (1982) para outros scelionídeos coletados em campo, parasitando ovos de tamanho semelhante e, também, com Orr et al. (1986), em levantamento de parasitóides de pentatomídeos, em campos de soja, como Podisus maculiventris (Say). Orr \& Boethel (1990) encontraram uma relação sexual de 1:4,4 para T. podisi, próxima a obtida neste estudo. No entanto, esses autores observaram uma relação de 1:15,8 para Telenomus cristatus Johnson, demonstrando haver grande variação deste parâmetro, que pode estar relacionada às condições de criação do parasitóide, hospedeiro e influência da temperatura.

A relação entre temperatura e desenvolvimento para $T$. brochymenae e $T$. podisi foi semelhante à obtida com $T$. euschisti (Ashmead) (Yeargan 1983),
Trissolcus oenone Dodd (James \& Warren 1991) e Telenomus cristatus (Yeargan 1980), as quais requerem um incremento térmico de 139 a 142 graus dias. T. podisi e $T$. brochymenae desenvolveram-se de 17 a $32^{\circ} \mathrm{C}$, apresentando temperatura base (tb) de 11,1 e 10,4 e, de 9,5 e $9,9^{\circ} \mathrm{C}$ para fêmeas e machos, respectivamente (Tabela 2). A constante térmica foi de 205,3 e 177,6 e, de 214,7 e 189,2 graus dias, respectivamente, para fêmeas e machos de $T$. podisi e $T$. brochymenae (Tabela 2). Observou-se pequena variação $\left(<1^{\circ} \mathrm{C}\right)$ entre as tb para fêmeas e machos da mesma espécie, entretanto, T. podisi apresentou tb maior que T. brochymenae em 1,6 e $0,5^{\circ} \mathrm{C}$, para fêmeas e machos, consequentemente, menor exigência em graus dias para completar o ciclo. Assim, as variações na tb e K entre as duas espécies estudadas, bem como para outras espécies, como T. oenone, que apresentou tb de $12,4^{\circ} \mathrm{C}$ e $\mathrm{K}$ de 165,5 graus dias (James \& Warren 1991), podem ser consideradas relativamente próximas, embora essas pequenas diferenças de tb e $\mathrm{K}$, podem caracterizar melhor seus efeitos, quando consideradas em estimativas de desenvolvimento (número de gerações) por um longo período, quando a temperatura pode tornar-se fator limitante durante um determinado tempo.

Cividanes \& Figueiredo (1996), estudando o desenvolvimento de $T$. podisi e $T$. 
brochymenae parasitando o pentatomídeo Piezodorus guildinii (Westwood), em temperaturas de 21 a $30^{\circ} \mathrm{C}$, observaram viabilidade de 81,6 e $62,2 \%$ a $21^{\circ} \mathrm{C}$ e de 38,6 e $38,8 \%$ a $30^{\circ} \mathrm{C}$, bem como tb de 11,2 e $14,1^{\circ} \mathrm{C}$, respectivamente. Portanto, estes resultados foram relativamente inferiores quanto à viabilidade e superiores quanto as tb, em relação aos obtidos sobre $P$. nigrispinus no presente estudo (Tabelas 1 e 2). Isto pode indicar P. nigrispinus como hospedeiro potencial desses parasitóides, embora a adequação do parasitóide ao hospedeiro envolva vários fatores químicos e físicos (Vinson 1985).

Avaliando-se temperaturas médias de cinco anos, em três regiões de cerrado de Minas Gerais com plantações de eucalipto e liberações de $P$. nigrispinus, considerando as exigências térmicas (tb) desse predador, determinadas por Didonet et al. (1996), com temperaturas de 18 a $35^{\circ} \mathrm{C}$, estimou-se um número de gerações de 10,2 por ano (Fig. 3A) e de 0,6 a 1,1 por mês (Fig. 3B). Nessas mesmas condições, podem ocorrer, respectivamente, 22,6 e 23,9 gerações de $T$. podisi e $T$. brochymenae, com variações mensais de 1,3 a 2,2 e 1,1 a 2,3 entre os meses mais frios e quentes do ano (Fig. 3B). O número de gerações anuais de $T$. podisi e $T$. brochymenae é, respectivamente, de 2,2 e 2,3 vezes maior que a de $P$. nigrispinus, o que pode ser um dos fatores que permite a esses parasitóides atingirem altas populações em final de surto de lagartas (Torres et al. 1996b). Nestas condições, $P$. nigrispinus apresenta populações elevadas, permitindo a manutenção da população dos parasitóides, como observado para $P$. sculptus Distant e os parasitóides de ovos Telenomus persimilis Ashmead e Ooencyrtus submetallicus (Howard) (Torres \& Zanuncio, dados não publicados), bem como para o complexo de pentatomídeos associados à soja (CorrêaFerreira \& Moscardi 1995). A utilização de vários hospedeiros predadores ou fitófagos e

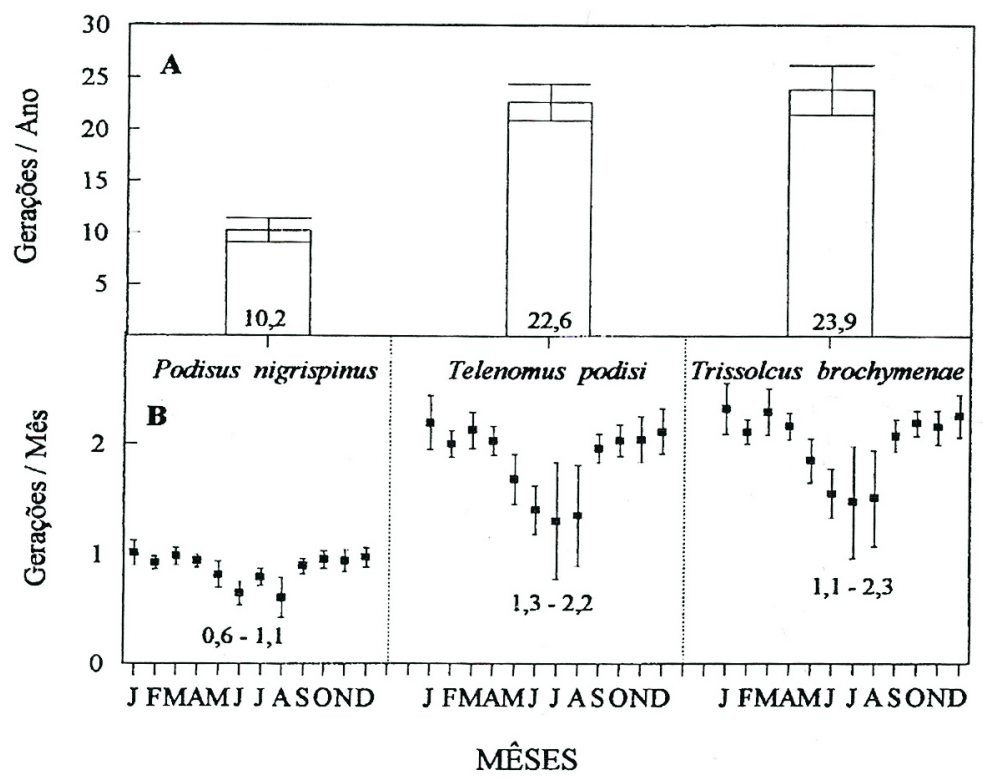

Figura 3. Número médio provável de gerações por ano (A) e por mês (B) de Podisus nigrispinus e de Telenomus podisi e Trissolcus brochymenae, em três áreas de reflorestamento com eucalipto. 
o rápido crescimento populacional, tornam estes parasitóides agentes eficientes de controle biológico.

A contínua variação de temperatura diurna e noturna na região do cerrado, onde dificilmente atinge nível inferior a tb para as espécies estudadas, mas frequentemente acima de $32^{\circ} \mathrm{C}$ (Tabela 2), pode resultar em taxas de desenvolvimento superiores ou viabilidade inferior as obtidas em laboratório, especialmente para T. podisi. Entretanto, pelos resultados obtidos, $T$. podisi, T. brochymenae e $P$. nigrispinus potencialmente são capazes de se desenvolverem durante todo o ano nas condições de campo (Fig. 3), nessa região. Portanto, o número de gerações desses parasitóides indica que possuem grande potencial de impacto sobre populações de $P$. nigrispinus, mas, outras variáveis devem ser consideradas. Estas podem incluir não somente o potencial reprodutivo, mas também a habilidade de procura e preferência de habitat, afetando a resposta funcional e numérica nas condições de campo, devido à heterogeneidade das áreas com eucalipto, comparado a ecossistemas agrícolas.

\section{Agradecimentos}

Ao CNPq e a CAPES pelos auxílios concedidos e a M. G. Soares pelo auxílio na manutenção das colônias de parasitóides e predadores.

\section{Literatura Citada}

Barcelos, J.A.V., J.C. Zanuncio, A.C. Oliveira \& E.C. Nascimento. 1994. Performance em duas dietas e descrição dos adultos de Brontocoris tabidus (Signoret) (Heteroptera: Pentatomidae). An. Soc. Entomol. Brasil 23: 519-524.

Campell, A., B.D. Frazier, N. Gilbert, A.P. Gutierrez \& M. Mackauer. 1974. Temperature requirements of some aphids and their parasites. J. Appl. Ecol. 11: 431438.
Cividanes, F.J. \& J.G. Figueiredo. 1996. Desenvolvimento e emergência de Trissolcus brochymenae (Ashmead) e Telenomus podisi Ashmead (Hymenoptera: Scelionidae) em diferentes temperaturas. An. Soc. Entomol. Brasil 25: 207-211.

Corrêa-Ferreira, B.S. \& F. Moscardi. 1995. Seasonal occurrence and host spectrum of egg parasitoids associated with soybean stink bugs. Biol. Control 5:196202.

Didonet, J., J.C. Zanuncio, M.C. Picanço \& C.S. Sediyama. 1996. Determinação das exigências térmicas de Podisus nigrispinus (Dallas, 1851) e de Supputius cincticeps Stal, 1860 (Heteroptera: Pentatomidae), em condições controladas. Rev. bras. Entomol. 40:61-63.

Frazer, B.D. \& R.R. McGregor. 1992. Temperature-dependent survival and hatching rate of eggs of seven species of Coccinelidae. Can. Entomol. 124: 305312 .

Higley, L.G., L.P. Pedigo \& K.R. Ostlie. 1986. Degray: a program for calculation degree-days, and assumptions behind the degree-day approach. Environ. Entomol. 15: 999-1016.

James, D.G. \& G.N. Warren. 1991. Effect of temperature on development, survival, longevity and fecundity of Trissolcus oenone Dodd (Hymenoptera: Scelionidae). J. Aust. Entomol. Soc. 30: 303-306.

Jubb, G.L. \& T.F. Watson. 1971. Development of the egg parasite Telenomus utahensis in two pentatomid hosts in relation to temperature and host age. Ann. Entomol. Soc. Am. 64: 202-205.

Miller, J.C. 1992. Temperature-dependent development of the convergent lady beetle (Coleoptera: Coccinelidae). 
Environ. Entomol. 21:197-201.

Morril, A.W. 1907. Description of a new species of Telenomus with observations on its habitats and life history. Amer. Nat. 41: 417-430.

O'Neil, R.J. \& J.L. Stimac. 1988. Measurement and analysis of arthropod predation on velvetbean caterpillar, Anticarsia gemmatalis Hübner, in soybean. Environ. Entomol. 17: 987-993.

Orr, D.B. \& D.J. Boethel. 1990. Reproductive potencial of Telenomus cristatus and T. podisi (Hymenoptera: Scelionidae), two egg parasitoids of pentatomids (Heteroptera). Ann. Entomol. Soc. Am. 83: 902-905.

Orr, D.B., J.S. Russin, D.J. Boethel \& W.A. Jones. 1986. Stinkbug (Hemiptera: Pentatomidae) egg parasitism in Louisiana soybean. Environ. Entomol. $15: 1250-1254$.

Torres, J.B., J.C. Zanuncio \& T.V. Zanuncio. 1996a. Produção e uso de percevejos predadores (Pentatomidae, Asopinae) no controle biológico de lagartas desfolhadoras, p. 41-51. In Workshop sobre Fitossanidade Florestal do Mercosul, 1, Santa Maria, Centro de Pesquisas Florestais, 80p.

Torres, J.B., J.C. Zanuncio, P.R. Cecon \& W.L. Gasperazzo. 1996b. Parasitismo de ovos de Podisus nigrispinus (Dallas) em áreas de liberação. An. Soc. Entomol. Brasil. 25: 465-473.

Torres, J.B., J.C. Zanuncio, M.C. Picanço \& A.C. Oliveira. 1996c. Parámetros poblacionales de tres parasitoides
(Hymenoptera: Scelionidae, Encyrtidae) utilizando al depredador Podisus nigrispinus (Hemiptera: Pentatomidae). Rev. Biol. Trop. 40: 233-240.

Tostowark, W. 1972. The effect of prey defense on the functional response of Podisus modestus (Hemiptera: Pentatomidae) to densities of the sawflies Neodiprion swainei and N. prati banksiane (Hymenoptera: Neodiprionidae). Can. Entomol. 104: 61-69.

Vinson, S.B. 1985. The behavior of parasitoids, p. 447-469. In:. G. A. Kerkut, and L. I. Gilbert (eds.), Comprehensive Insect Physiology Biochemistry and Pharmacology, vol. 4. New York, Pergamon Press, 529p.

Waage, J.K. 1982. Sib-mating and sex ratio strategies in scelionid wasps. Ecol. Entomol. 7: 103-112.

Yeargan, K.V. 1980. Effects of temperature on developmental rate of Telenomus podisi (Hymenoptera: Scelionidae). Ann. Entomol. Soc. Am. 73: 339-342.

Yeargan, K.V. 1983. Effects of temperature on developmental rate of Trissolcus euschisti (Hymenoptera: Scelionidae), a parasite of stink bug eggs. Ann. Entomol. Soc. Am. 76: 757-760.

Zanuncio, J.C., J.B. Alves, T.V. Zanuncio \& J.F. Garcia. 1994. Hemipterous predators of eucalypt defoliator caterpillars. For. Ecol. Manag. 65: 65-73.

Recebido em 04/09/96. Aceito em 12/09/97. 